Berg Huettenmaenn Monatsh (2022) Vol. 167 (4): 134-139

https://doi.org/10.1007/s00501-022-01206-6

(C) The Author(s), under exclusive licence to Austrian Society for Metallurgy of Metals (ASMET) and Bergmännischer Verband Österreich (BVÖ) 2022

\title{
Der österreichische Bergbau: Nationale und internationale Aspekte
}

\author{
Thomas Spörker' ${ }^{1}$ Susanne Strobl'2, Robert Holnsteiner ${ }^{2}$ und Christian Reichl²
}

\begin{abstract}
${ }^{1}$ Abteilung IV/6 Bergbau - Technik und Sicherheit, Sektion IV Telekommunikation, Post und Bergbau, Bundesministerium für Landwirtschaft, Regionen und Tourismus, Wien, Österreich ${ }^{2}$ Abteilung IV/5 Mineralrohstoffpolitik, Sektion IV Telekommunikation, Post und Bergbau, Bundesministerium für Landwirtschaft, Regionen und Tourismus, Wien, Österreich
\end{abstract}

Eingegangen 24. Januar 2022; angenommen 31. Januar 2022; online publiziert 23. Februar 2022

\begin{abstract}
Zusammenfassung: Die bedarfsgerechte Versorgung mit mineralischen Rohstoffen ist eine unverzichtbare Grundlage für eine erfolgreiche Wirtschaft. Um die Versorgungssicherheit gewährleisten und auf aktuelle Herausforderungen reagieren zu können, hat das Bundesministerium für Landwirtschaft, Regionen und Tourismus Ende 2021 den „Masterplan Rohstoffe 2030" veröffentlicht. Innovative Schlüsseltechnologien, die unsere Zukunftsfähigkeit sichern und zur Zielerreichung des Europäischen Green Deals beitragen sollen, sind unmittelbar mit dem Einsatz von Rohstoffen verbunden. Die Produktionsstatistik spiegelt diesen steigenden Rohstoffbedarf bereits wider. So ist die weltweite Bergbauproduktion seit dem Jahr 2000 um fast $60 \%$ auf ein Allzeithoch von rund $17,9 \mathrm{Mrd}$. Tonnen im Jahr 2019 gestiegen. Bemerkenswert ist, dass Europa der einzige Kontinent ist, wo in den letzten zwanzig Jahren die Rohstoffproduktion zurückgegangen ist. Aller Voraussicht nach wird im Jahr 2020 Ozeanien, vor allem Australien, mehr Rohstoffe produzieren als Europa. Im vergangenen Jahr sind die Rohstoffpreise teilweise stark gestiegen und die steigende Nachfrage für Zukunftstechnologie-Rohstoffe lässt weitere Preissteigerungen erwarten. Das Ziel der Europäischen Union ist der Aufbau und die Stärkung der Resilienz der heimischen Wirtschaft unter anderem durch die Förderung des europäischen Bergbaus und der Kreislaufwirtschaft sowie der Diversifizierung von Lieferketten
\end{abstract}

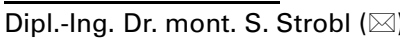

Abteilung IV/5 Mineralrohstoffpolitik, Sektion IV

Telekommunikation, Post und Bergbau,

Bundesministerium für Landwirtschaft, Regionen und Tourismus, Denisgasse 31,

1200 Wien, Österreich

susanne.strobl@bmlrt.gv.at unter steter Einhaltung der Vorgaben der Sustainable Development Goals.

Schlüsselwörter: Masterplan Rohstoffe,

Rohstoffproduktion, Konfliktminerale, Rohstoffpreise, Rohstoffpolitik

National and International Aspects of Austrian Mining

Abstract: The security of supply of mineral raw materials is an indispensable basis for a flourishing economy. In order to ensure this and to be able to react to current challenges, the Austrian Federal Ministry of Agriculture, Regions and Tourism published its raw materials strategy called "Masterplan Mineral Resources 2030" at the end of 2021. The goals of the European Green Deal can be achieved with innovative key technologies that are directly linked with the input of raw materials. The increasing demand for mineral resources is already reflected by production statistics. The world mining production has increased by almost $60 \%$ since 2000 to an all-time high of around 17.9 billion tonnes in 2019. It is remarkable that Europe is the only continent with a declining production rate over the last twenty years. It is very likely that Oceania, especially Australia, will produce more mineral raw materials than Europe in 2020. Most commodity prices have increased significantly in the last year and the growing demand for the use of resources in future technologies will probably lead to high commodity prices in the future. The aim of the European Union is to build and strengthen the resilience of its economy by promoting mining activities and circular economy as well as the diversification of supply chains while always respecting the terms of the Sustainable Development Goals. 
Keywords: Raw materials strategy, Minerals production, Conflict minerals, Commodity prices, Mineral resources policy, Nationale Aspekte

\section{Nationale Aspekte}

\subsection{Masterplan Rohstoffe 2030}

Die Sicherstellung einer umfassenden und nachhaltigen Rohstoffversorgung, innerhalb derer die heimische Ressourcenbasis gestärkt und die internationale Beschaffung gewährleistet werden, ist unumgänglich, wenn man den Fortschritt vorantreiben und den Wohlstand innerhalb der österreichischen Bevölkerung erhalten möchte. Um dies gewährleisten und gleichzeitig die Resilienz des heimischen Rohstoffsektors stärken zu können, hat das Bundesministerium für Landwirtschaft, Regionen und Tourismus den Masterplan Rohstoffe 2030 [1] als Rohstoffstrategie erarbeitet und Ende 2021 veröffentlicht (Download frei verfügbar unter https://info.bmlrt.gv.at/service/publikationen/ bergbau/masterplan-rohstoffe-2030.html).

Die ausreichende Versorgung mit mineralischen Rohstoffen zu fairen Marktbedingungen ist eine wesentliche Grundlage für eine wirtschaftliche Entwicklung. Umstände wie Pandemien oder Naturkatastrophen sowie geopolitische oder wirtschaftliche Ereignisse können zu einer Verknappung von mineralischen Ressourcen führen. Die anhaltende Coronavirus-Pandemie führt uns die Verwundbarkeit in einer globalisierten Welt mit komplexen Abhängigkeiten und weit verzweigten Wertschöpfungsketten schonungslos vor Augen. Sie offenbart Fragen nach der Versorgungssicherheit für Produkte des täglichen Bedarfs, aber auch für Grundstoffe, die für die weiterverarbeitende Industrie dringend benötigt werden. Der Masterplan Rohstoffe liefert Lösungsvorschläge, um auf die Herausforderungen der jetzigen Dekade entsprechend reagieren zu können, denn die Klima-, Energie- und Industriewende bedeutet gleichzeitig auch eine Rohstoffwende.

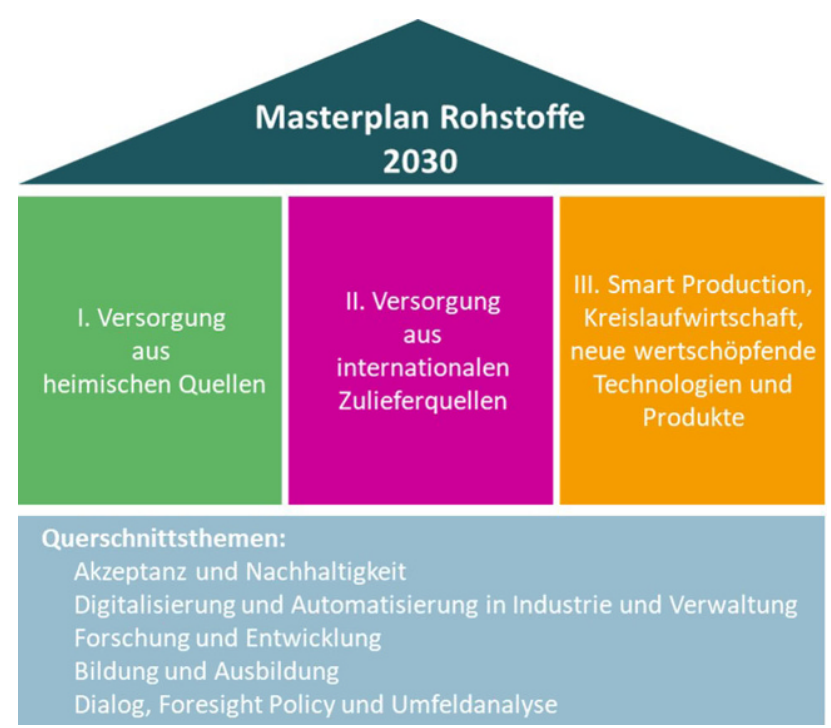

Abb. 1: Struktur des Masterplans Rohstoffe 2030 [1]
Der Masterplan Rohstoffe 2030 behandelt die für Österreich relevanten rohstoffpolitischen Dimensionen:

- Versorgung aus heimischen Quellen,

- Versorgung aus internationalen Zulieferquellen und

- intelligente Produktion, Kreislaufwirtschaft sowie neue wertschöpfende Technologien und Produkte.

Wegbegleitend wird der Umgang mit den Themen Nachhaltigkeit, Akzeptanz, Digitalisierung, Innovation, Bildung und Dialog beschrieben (Abb. 1).

Dieser Masterplan ist am Rohstoffkreislauf für primäre und sekundäre mineralische Rohstoffe orientiert und legt mit einem umfangreichen Maßnahmenpaket den Grundstein für eine resiliente Entwicklung Österreichs.

\subsection{Rohstoffproduktion in Österreich}

Im Jahr 2018 wies Österreich einen Ressourceneinsatz von 167 Mio.t auf. Dies entspricht in etwa 19t pro Kopf und Jahr. Rund drei Viertel davon sind mineralische Rohstoffe [2], die den Grundbedarf vielerlei Branchen und Bedürfnisse abdecken. Aufgrund dessen tragen Roh- und Grundstoffe zur Generierung von etwa $25 \%$ des österreichischen Bruttoinlandsproduktes bei. Rund eine Million Erwerbstätige sind hierzulande mit der Gewinnung und Weiterverarbeitung von Rohstoffen beschäftigt [3].

Die jährliche Produktion mineralischer Rohstoffe in Österreich lag im Jahr 2020 bei rund 79,6 Mio.t (im Jahr 2019 rund 80,0 Mio.t), wobei diese Zahlen die gem. § 178 Abs. 3 MinroG gemeldeten und statistisch erfassten Daten beschreiben [4].

Im Jahr 2020 stellten mit ca. 52,0 Mio.t und somit fast zwei Drittel die grundeigenen mineralischen Rohstoffe den größten Anteil der heimischen Produktion dar, gefolgt von den bergfreien mineralischen Rohstoffen mit ca. 25,3 Mio.t (i.e. $32 \%$ der Gesamtproduktion) und den bundeseigenen mit 2,2 Mio.t (i.e. 3\% der Gesamtproduktion). Die Gewinnung der grundeigenen und bundeseigenen mineralischen Rohstoffe sinkt seit dem Jahr 2018, wohingegen die der bergfreien steigt. Die Produktion von Erdöl und Erdgas ist im Jahr 2020 von rund 1,4 Mio.t oil equ. (2019) auf etwa 1,2 Mio.t oil equ. gesunken [4].

Bemerkenswert ist, dass Österreich bei einer Reihe von Rohstoffen im internationalen Vergleich auf eine hervorragende Produktionsbasis verweisen kann. So ist Österreich der jeweils siebtgrößte Produzent von Wolfram und Magnesit weltweit im Jahr 2019 [5].

\subsection{Unfallstatistik}

In der Sparte der mineralrohstoffgewinnenden Industrie ereigneten sich im Jahr 2020 insgesamt 140 Unfälle. Dies bedeutet gegenüber dem Jahr 2019 mit 153 Unfällen einen Rückgang der Unfallanzahl von $8,5 \%$. Von den Unfällen im Jahr 2020 waren 39 schwerer und 99 leichter Natur. Leider waren auch zwei tödliche Unfälle zu beklagen [6]. 


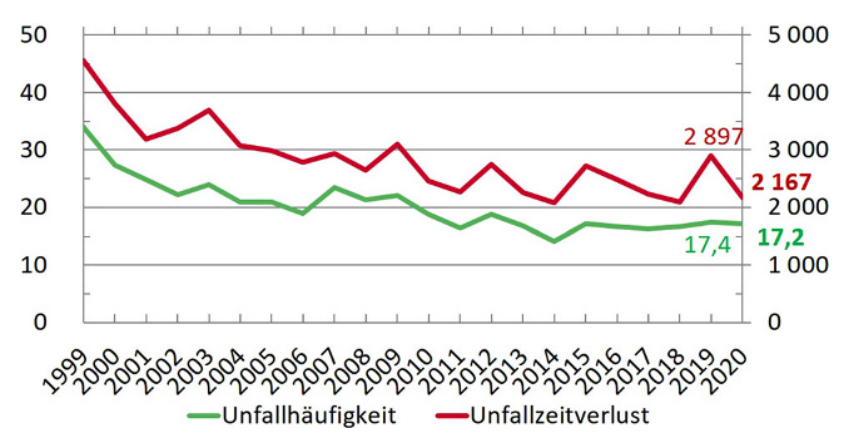

Abb. 2: Entwicklung Unfallhäufigkeit und Unfallzeitverlust [6]

Die Anzahl der verfahrenen Stunden ist im Jahr 2020 mit rund 8,1 Mio. um 7,2\% gesunken. Im Jahr 2020 betrug die Unfallhäufigkeit, das ist die Zahl der Unfälle je 1 Mio. verfahrener Stunden, 17,2 (Abb. 2). Im Vergleich zum Jahr 2019 bedeutet dies einen Rückgang um 1,1\%. Durch meldepflichtige Arbeitsunfälle sind im österreichischen Bergbau im Jahre 2020 insgesamt $17.640 \mathrm{~h}$ - das sind um 30,6\% weniger an Arbeitszeit als im Vorjahr - entgangen. Der Unfallzeitverlust, das ist die Zahl der durch Unfälle entgangenen Stunden je 1 Mio. verfahrener Stunden und somit die Kennzahl für die Schwere der Unfälle, reduzierte sich im Vergleich zum Vorjahr um 25,2\% auf 2167 (Abb. 2). Die durchschnittliche Heildauer eines Unfalles belief sich im Jahr 2020 auf 126h [6].

\subsection{Konfliktminerale}

Die Verordnung (EU) 2017/821 zur Festlegung von Sorgfaltspflichten in der Lieferkette für Unionseinführer von Zinn, Tantal, Wolfram, deren Erzen und Gold aus Konflikt- und Hochrisikogebieten (kurz: Konfliktmineraleverordnung) [7] verpflichtet Unionseinführer, Risiken im Bereich ihrer Lieferketten zu identifizieren und geeignete Maßnahmen zu deren Minimierung zu treffen. Dadurch soll ein wesentlicher Beitrag zur Vermeidung der Finanzierung von Konflikten durch Gewinne aus dem Rohstoffabbau und -handel sowie der damit einhergehenden, schwerwie- genden Menschenrechtsverletzungen geleistet werden. Im Rahmen einer verantwortungsvollen Beschaffungspolitik hat der Unionseinführer seine Lieferkette entsprechend dem OECD-Leitfaden für die Erfüllung der Sorgfaltspflicht zur Förderung verantwortungsvoller Lieferketten für Minerale aus Konflikt- und Hochrisikogebieten [8] zu gestalten. Dieser umfasst unter anderem Risikomanagementpflichten, die Überprüfung der Unionseinführer durch unabhängige Dritte (sogenannte Audits) sowie Offenlegungspflichten hinsichtlich der Lieferkettenstrategien und des Risikomanagements.

Die Konfliktmineraleverordnung ist unmittelbar anwendbar. Durch die Novellierung des Mineralrohstoffgesetzes [9] wurden entsprechende Begleitbestimmungen geschaffen. Die Verpflichtungen für Unionseinführer gelten seit 1. Jänner 2021. Die nachträglichen Kontrollen durch die zuständige Behörde beginnen 2022 und umfassen die Importe des Jahres 2021. Die Abteilung Mineralrohstoffpolitik wurde von der Bundesministerin für Landwirtschaft, Regionen und Tourismus mit der unmittelbaren Durchführung der Konfliktmineraleverordnung betraut.

Unter https://info.bmlrt.gv.at/themen/bergbau/konflikt mineraleverordnung.html finden Sie nähere Informationen zur Umsetzung der Konfliktmineraleverordnung in Österreich.

\section{Internationale Aspekte}

\subsection{Globale Rohstoffproduktion im Jahr 2019}

Die Weltbergbauproduktion hat im Jahr 2019 mit rund $17,9 \mathrm{Mrd} . \mathrm{t}$ ihr Allzeithoch erreicht und ist somit seit der Jahrtausendwende um 58,7\% gestiegen. Die Produktionszahlen von Baurohstoffen werden in der Publikation "World Mining Data" nicht abgebildet, da diese auf den Weltmärkten nicht gehandelt werden bzw. keine authentischen und somit vergleichbaren Zahlen vorliegen. Der erstmalige stärkere Produktionsrückgang seit 2009 bei den Energierohstoffen mit -3,1\% zwischen 2015 und 2016 wurde durch Produktionssteigerungen in den Folgejahren 2017 bis 2019 wieder relativiert $(+3,0 \%,+3,2 \%,+1,2 \%)$.
Abb. 3: Weltbergbauproduktion nach Rohstoffgruppen, in Mio. metr.t, exkl. Baurohstoffe. (Nach [5])

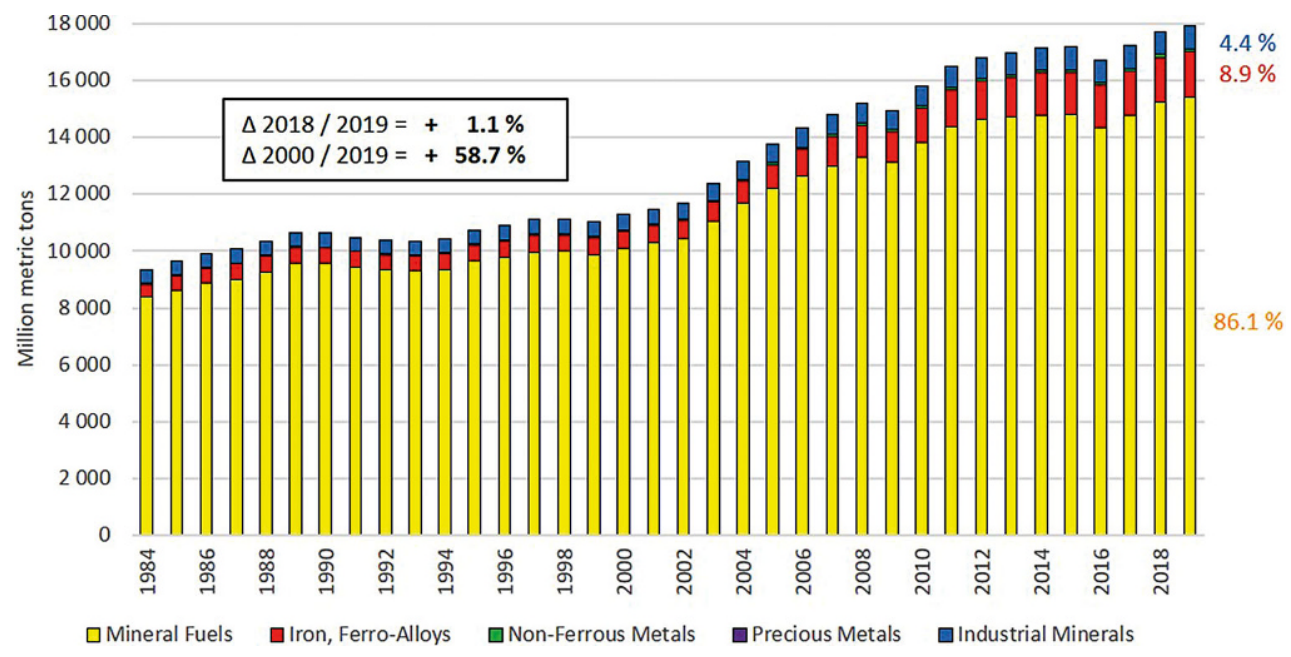




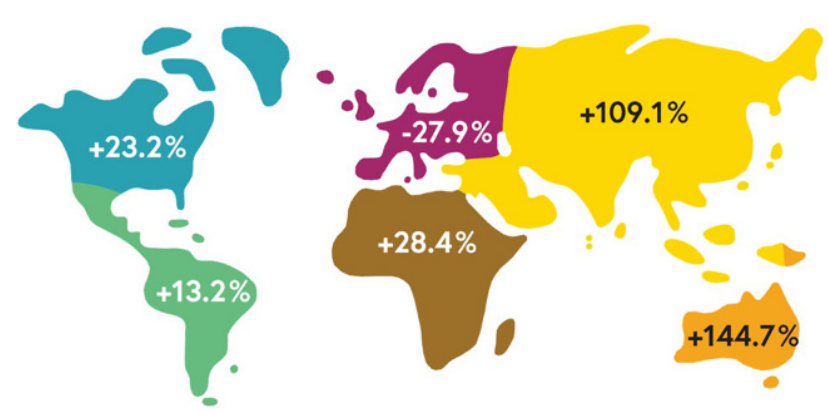

Abb. 4: Zeitliche Veränderung der Bergbauproduktion je Kontinent seit 2000, exkl. Baurohstoffe. (Nach [5])

Ausschlaggebend für diese Steigerungen ist die Erdöl- und Erdgasförderung in den USA bzw. die chinesische Kohleförderung. Der erstmalige Produktionsrückgang in 2019 in der Gruppe der Nichteisenmetalle ist auf eine Reduktion der Produktion von primärem Aluminium in China zurückzuführen. Mengenmäßig überwiegen die Energierohstoffe an der Weltbergbauproduktion mit ca. $86,1 \%$, gefolgt von Eisen und den Stahlveredlern mit ca. 8,9\% und den Industriemineralen mit ca. 4,4\% (Abb. 3). Die Produktionsmengen in den Gruppen Nichteisenmetalle und Edelmetalle sind gegenüber den anderen Rohstoffgruppen zu gering um in Abb. 3 erkennbar aufzuscheinen [5].

Abb. 4 zeigt in einer vereinfachten Weltkarte die gewaltigen Zuwachsraten der Bergbauproduktion seit 2000 in Ozeanien und Asien. Als einziger Kontinent weist Europa seit fast zwei Dekaden eine abnehmende Tendenz in der Bergbauproduktion auf. Die größten fünf Produzentenländer sind China, USA, Russland, Australien und Indien, wobei China mit rund 4,324 Mrd.t fast ein Viertel der gesamten Weltbergbauproduktion einnimmt und bei insgesamt 32 mineralischen Rohstoffen Top-Produzent ist. Norwegen liegt als erstes europäisches Land mit einem Produktionsvolumen von rd. 174,5 Mio.t an 16. Stelle, gefolgt von Deutschland [5].

Mit Ausnahme von Europa $(-10,7 \%)$ und Lateinamerika $(-6,5 \%)$ konnten auf allen anderen Kontinenten von 2018 auf 2019 Steigerungsraten der Rohstoffproduktion erzielt werden (Nordamerika $+3,8 \%$, Afrika $+0,4 \%$ und Ozeanien $+3,9 \%$ ). Ozeanien (im Wesentlichen Australien) produziert seit 2013 größere Mengen an Rohstoffen als der vermeintliche Rohstoffkontinent Afrika, seit 2016 größere Mengen an Rohstoffen als Lateinamerika und wird aller Voraussicht nach noch im Jahr 2020 die rückläufige Rohstoffproduktion Europas überholen (Abb. 5). Der Grund für den deutlichen Anstieg der Rohstoffproduktion der letzten Jahre in Nordamerika liegt in einem starken Anstieg der US-amerikanischen Erdöl-, Naturgas- und Kohleproduktion. Der weitere Rückgang der Rohstoffproduktion in Lateinamerika in 2019 um rd. 6,5\% ist auf die verminderte venezolanische Erdölproduktion zurückzuführen. Der Anteil der in 2019 abermals stärker rückläufigen europäischen Bergbauproduktion an der Weltbergbauproduktion liegt bei rund $7,1 \%(8,0 \%$ in 2018). Diese Abnahme der Rohstoffproduktion Europas ist auf die rückläufige Produktion an Energierohstoffen zurückzuführen, u. a. auf das Ende der deutschen und spanischen Steinkohleproduktion [5].

\subsection{Preisentwicklungen}

\subsection{1 Öl- und Gaspreise}

Seit dem letzten Jahr sind die Energiepreise stark gestiegen. Dieser große Anstieg betrifft alle und beeinträchtigt die Wettbewerbsfähigkeit der im globalen Wettbewerb stehenden Unternehmen am meisten, wobei insbesondere die Entwicklung des Gaspreises der energieintensiven Industrie Sorgen bereitet. Die weltweite Nachfrage nach Öl und Gas ist aufgrund der wirtschaftlichen Erholung gestiegen und ist hauptsächlich für das aktuelle Preishoch verantwortlich. Die Europäische Kommission zeigt in einer am 13. Oktober 2021 veröffentlichten Mitteilung zu den steigenden Energiepreisen [10] auf, welche Instrumente zur Verfügung stehen, um die Auswirkungen der hohen Energiepreise bewältigen und den Menschen und Unternehmen in Europa helfen zu können.
Abb. 5: Zeitlicher Verlauf (1984 bis 2019) der Weltbergbauproduktion aufgeteilt nach Kontinenten (exkl. Asien), exkl. Baurohstoffe. (Nach [5])

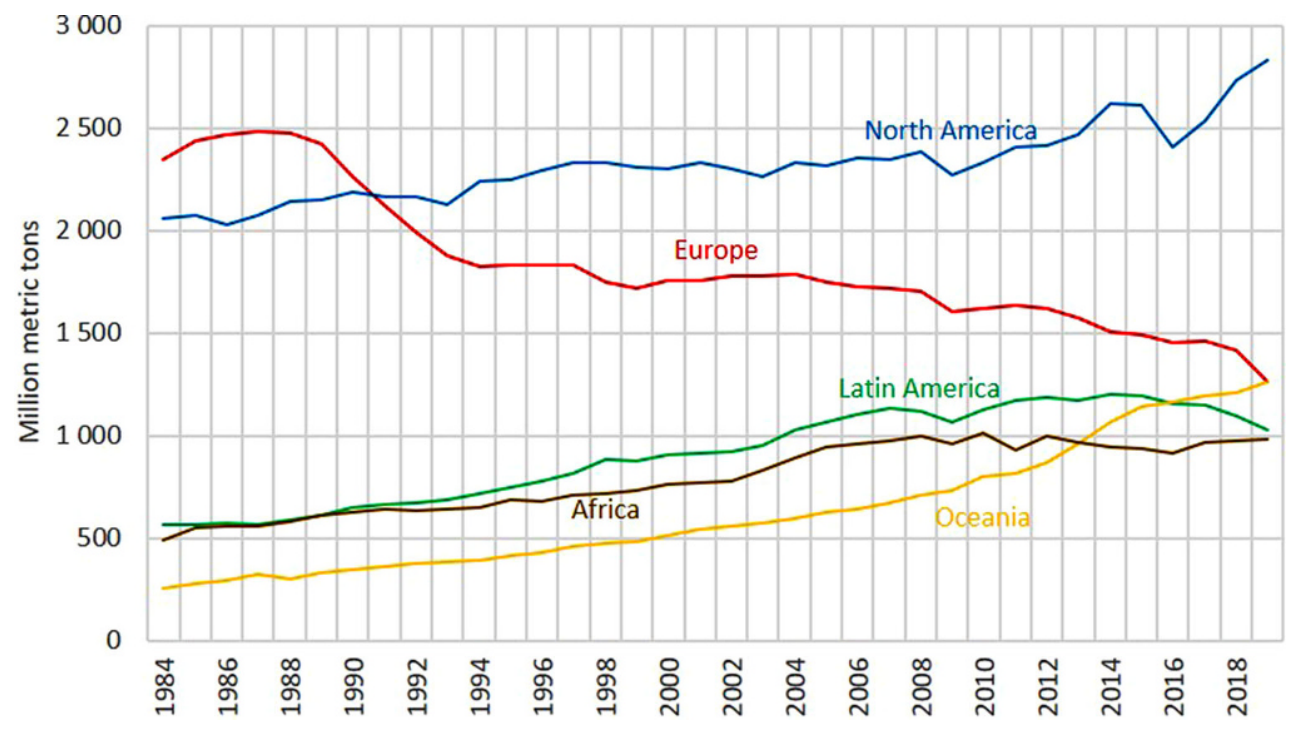




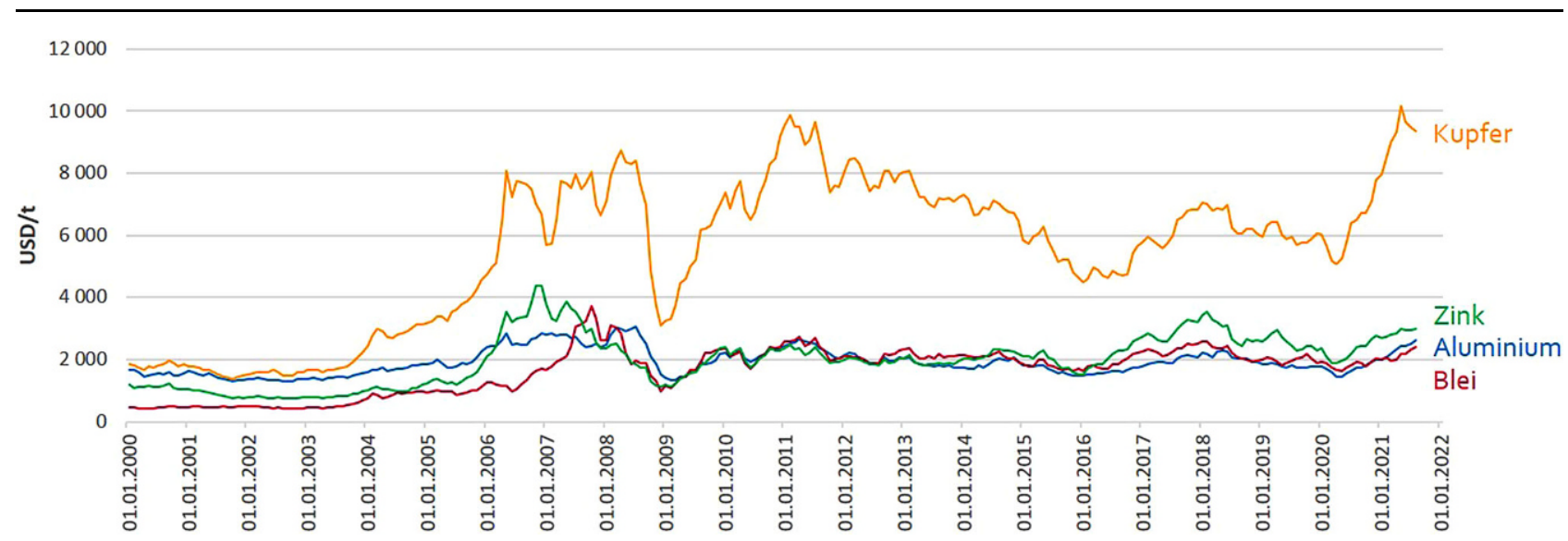

Abb. 6: Entwicklung der Buntmetallpreise seit dem Jahr 2000. (Nach [11])

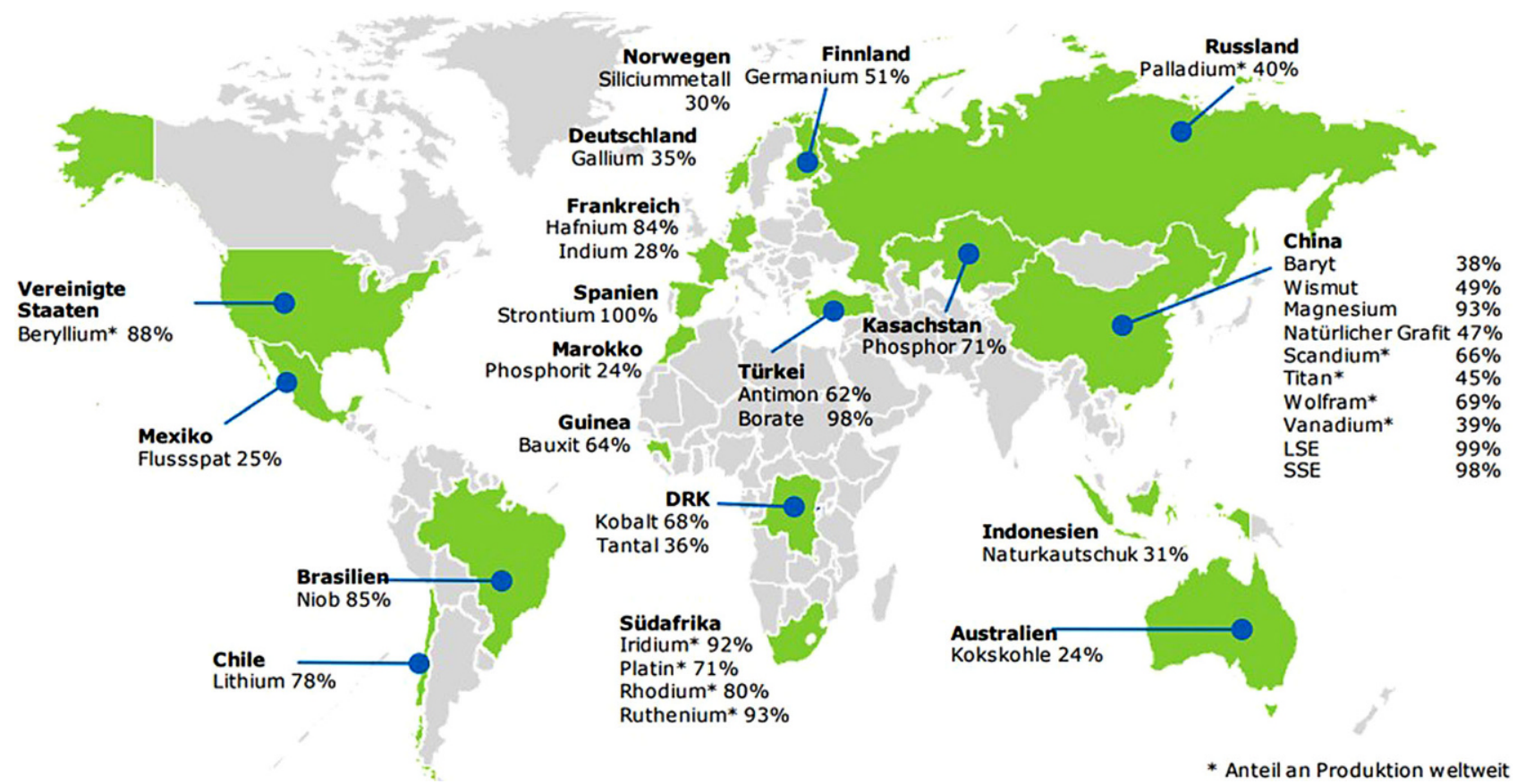

Abb. 7: Wichtigste Lieferländer von kritischen Rohstoffen an die EU. (Nach [13])

\subsubsection{Buntmetallpreise}

Das Anlaufen der Konjunktur nach den Einbrüchen durch die coronabedingten Lockdowns lässt auch die Preise für Buntmetalle wieder ansteigen. Der Kupferpreis hat im Sommer 2021 mit über 10.000 USD pro Tonne sein Zehn-Jahreshoch überschritten (Abb. 6).

Um die Preise stabilisieren zu können, verkaufte China Anfang Oktober 2021 fast eine Million Tonnen Buntmetalle aus staatlichen Reserven. Zuvor wurden infolge von Energiebeschränkungen die Produktionsanlagen in vielen chinesischen Provinzen vorübergehend stillgelegt. Dennoch liegen die Preise nach wie vor deutlich oberhalb des Preisniveaus vor Beginn der chinesischen Produktionsbeschränkungen [12].

Der Preis des Schlüsselrohstoffs Eisenerz ist nach einem starken Hoch im Sommer 2021 von über 200 USD pro Tonne wieder auf ca. 120 USD pro Tonne gesunken.
Eine steigende Nachfrage nach Rohstoffen für die benötigten Technologien, damit die "Grüne Wende" gelingen kann, lässt weitere Preissteigerungen bei den dafür erforderlichen Rohstoffen erwarten.

\subsection{Entwicklungen auf EU-Ebene}

Die Europäische Kommission veröffentlicht alle drei Jahre eine Liste der kritischen Rohstoffe für die EU. Die "Mitteilung zur Widerstandsfähigkeit der EU bei kritischen Rohstoffen" [13] vom Herbst 2020 definiert 30 Rohstoffe als kritisch, also volkswirtschaftlich bedeutend und potenziellen Lieferengpässen unterworfen. Abb. 7 stellt die wichtigsten Lieferländer von kritischen Rohstoffen an die EU dar. Bemerkenswert ist die Abhängigkeit von Selten-Erd-Metallen (LSE: leichte, SSE: schwere) aus China. 
Der mit dieser Mitteilung [13] im Zusammenhang stehende Aktionsplan beinhaltet die Gründung einer Europäischen Rohstoffallianz (ERMA). Diese hat es sich zum Ziel gesetzt, Europa wirtschaftlich widerstandsfähiger zu machen, indem sie seine Lieferketten diversifiziert, Arbeitsplätze schafft, Investitionen ermöglicht, Innovationen fördert, junge Talente ausbildet und zu den weltweit besten Rahmenbedingungen für Rohstoffe und Kreislaufwirtschaft beiträgt. Die ERMA stellt ein internationales Netzwerk von Stakeholdern aus privaten und öffentlichen Bereichen dar. Das erste Ziel der Allianz ist der Aufbau der Resilienz und offenen strategischen Autonomie für die Wertschöpfungsketten von Selten-Erd-Magneten und Motoren, bevor diese auf andere Rohstoffbereiche erweitert werden soll [14].

Ein weiterer wesentlicher Bestandteil des Aktionsplans für kritische Rohstoffe ist die Stärkung der nachhaltigen und verantwortungsvollen Beschaffung und Verarbeitung von Rohstoffen in der EU, in der die öffentliche Akzeptanz einen wesentlichen Aspekt darstellt. Dieses Ziel wird in den „EU-Grundsätzen für nachhaltige Rohstoffe " [15] unterstützt, welche im Juli 2021 veröffentlicht worden sind. Das Ziel dieser Grundsätze besteht darin, das Verständnis der Mitgliedstaaten in Bezug auf die nachhaltige Rohstoffgewinnung (von der Exploration bis nach der Stilllegung) und Verarbeitungstätigkeiten in der EU anzugleichen und die allgemeine Ausrichtung zur Erreichung der Sustainable Development Goals festzulegen.

\section{Literatur}

1. Bundesministerium für Landwirtschaft, Regionen und Tourismus: Masterplan Rohstoffe 2030. , Wien (2021)

2. Bundesministerium für Landwirtschaft, Regionen und Tourismus, Bundesministerium für Klimaschutz, Umwelt, Energie, Mobilität, Innovation und Technologie: Ressourcennutzung in Österreich 2020 Bd. 3. Wien (2020)

3. Statistik Austria: Konjunkturerhebung (2020)
4. Bundesministerium für Landwirtschaft, Regionen und Tourismus: Österreichisches Montanhandbuch 2021 Bd. 95. Wien (2021)

5. Bundesministerium für Landwirtschaft, Regionen und Tourismus: World Mining Data 2021 Bd. 36. Wien (2021)

6. Bundesministerium für Landwirtschaft, Regionen und Tourismus: Statistische Auswertung des Unfallgeschehens in der österreichischen mineralrohstoffgewinnenden Industrie im Jahr 2020. Wien (2021)

7. EU: Amtsblatt der Europäischen Union L 130, 19.05.2017, S. 1-20 Verordnung (EU) 2017/821 des Europäischen Parlaments und des Rates vom 17. Mai 2017 (2017). zur Festlegung von Pflichten zur Erfüllung der Sorgfaltspflichten in der Lieferkette für Unionseinführer von Zinn, Tantal, Wolfram, deren Erzen und Gold aus Konflikt- und Hochrisikogebieten

8. OECD: OECD-Leitfaden für die Erfüllung der Sorgfaltspflicht zur Förderung verantwortungsvoller Lieferketten für Minerale aus Konfliktund Hochrisikogebieten Bd. 3. OECD Publishing, Paris (2019)

9. BGBI. I Nr. 14/2021: Bundesgesetz, mit dem das Mineralrohstoffgesetz geändert wird MinroG-Novelle Konfliktminerale

10. COM: 660 final: Mitteilung der Kommission an das Europäische Parlament, den Europäischen Rat, den Rat, den Europäischen Wirtschafts- und Sozialausschuss und den Ausschuss der Regionen. Brüssel (2021). Steigende Energiepreise - eine „Toolbox" mit Gegenmaßnahmen und Hilfeleistungen

11. International Monetary Fund-IMF: International Monetary FundIMF. https://www.imf.org/en/Research/commodity-prices, Zugegriffen: 13. Jan. 2022

12. Deutsche Rohstoffagentur in der Bundesanstalt für Geowissenschaften und Rohstoffe (BGR): Rohstoff Preismonitor, November 2021. Berlin (2021)

13. COM: 474 final: Mitteilung der Kommission an das Europäische Parlament, den Rat, den Europäischen Wirtschafts- und Sozialausschuss und den Ausschuss der Regionen. Brüssel (2020). Widerstandsfähigkeit der EU bei kritischen Rohstoffen: Einen Pfad hin zu größerer Sicherheit und Nachhaltigkeit abstecken

14. European Raw Materials Alliance-ERMA: https://erma.eu, Zugegriffen: 3. Jan. 2022

15. Europäische Kommission: EU-Grundsätze für nachhaltige Rohstoffe. Amt für Veröffentlichungen der Europäischen Union, Luxemburg (2021)

Hinweis des Verlags. Der Verlag bleibt in Hinblick auf geografische Zuordnungen und Gebietsbezeichnungen in veröffentlichten Karten und Institutsadressen neutral. 